\title{
ARTERIAL BLOOD PRESSURE AND HEART RATE RESPONSE IN PATIENTS WITH ACUTE ISCHEMIC STROKE CORRELATION WITH THE SEVERITY OF CARDIOVASCULAR AUTONOMIC DYSFUNCTION
}

\author{
Darina Georgieva-Hristova ${ }^{1}$, Silva Andonova ${ }^{1}$, Evgeniya Kalevska ${ }^{1}$, Tihomir Drenski ${ }^{1}$, \\ Mila Boyadzhieva ${ }^{2}$
}

\author{
${ }^{1}$ Department of Neurology and Neuroscience, Medical University of Varna, Bulgaria \\ ${ }^{2}$ Department of Endocrinology and Metabolic disease, Medical University of Varna, Bulgaria
}

\begin{abstract}
BACKGROUND AND PURPOSE: Despite numerous studies, the mechanisms of blood pressure and heart rate regulation in cerebrovascular diseases remain incompletely understood. The aim of the study was to determine the changes in blood pressure and heart rate in patients with acute hemispheric ischemic stroke and to seek a relationship with the severity of the cardiovascular autonomic dysfunction.

METHODS: 20 healthy volunteers and 50 patients with acute hemispheric ischemic stroke participated in the study. The values of blood pressure and heart rate were registered. Cardiovascular autonomous disorders were examined using the Ewing test battery. The patients were divided into two groups according to the severity of the autonomous disorders.

RESULTS: Elevated BP values at admission were observed in $56 \%$ of our patients, and significantly higher values of systolic blood pressure $(p<0.001)$, diastolic blood pressure (DBP) $(p=0.001)$, and mean arterial pressure $(p<0.001)$, as well as of the heart rate $(p=0.027)$ were found, compared to the control group. The most significantly increased values of the indicators were reported in patients with a stroke in the territory of the right middle cerebral artery with an involvement of the insular cortex. The results of the t-test performed, showed significantly higher values of systolic blood pressure in patients with severe autonomic disorders $(\mathrm{t}=-\mathbf{2 . 1 4} ; \mathrm{p}=\mathbf{0 . 0 3 6})$.

CONCLUSION: A variability in BP and HR with a tendency to increase was observed in patients with acute hemispheric ischemic stroke (ICS) compared to controls, with the highest values reported in patients with ICS in the territory of right middle cerebral artery (TRMCA) with involvement of the insular cortex (IC). A positive correlation was found between the values of systolic blood pressure (SBP) and the severity of autonomic dysfunction (AD).
\end{abstract}

Keywords: cardiovascular autonomic dysfunction, stroke, blood pressure

Address for correspondence:

Darina Georgieva

Department of Neurology,

Medical University of Varna

55 Marin Drinov Str.

9002 Varna

e-mail:darina_georgieva@abv.bg

Received: February 21, 2016

Accepted: April 04, 2016

\section{INTRODUCTION}

Changes in heart rate (HR) and blood pressure (BP) regulation are among the most common disorders of cardiovascular autonomic regulation. A number of cortical-subcortical structures affect the hemodynamic regulation, influencing sympathetic and parasympathetic modulation $(1,2,3)$. 
Arterial blood pressure and heart rate response in patients with acute ischemic stroke correlation with the severity of cardiovascular ...

$\mathrm{BP}$ in healthy individuals is characterized by the so-called "dipping" model and shows a daily variation characterized by a decrease in BP by $10-20 \%$ at night (4). In patients with acute ischemic stroke, this model is disrupted ("non-dipping") (5), with pathophysiological mechanisms being complex and poorly clarified (6), the increased sympathetic activity playing an essential part in them (4).

BP's early variability affects brain perfusion $(7,8)$. Observations show that the clinical outcome in acute ischemic stroke depends not only on the initial BP but also on the direction and the rate of the change in BP in the first 24-48 hours (9). On the other hand, the rise of $\mathrm{BP}$ after stroke may represent a compensatory response to maintain adequate cerebral perfusion. In the acute phase of ischemic stroke, $\mathrm{BP}$ values vary widely and the clinical behavior is largely based on the mean values of $\mathrm{BP}$ at admission.

The aim of this study was to investigate the main hemodynamic parameters (HR and $\mathrm{BP}$ ) and to conduct a comprehensive research of the disorders in the cardiovascular autonomic function in patients with ICS. We also aimed at seeking a relationship between the values of $\mathrm{BP}$ and $\mathrm{HR}$ and the severity of the autonomic dysfunction.

\section{METHODS}

The study included 20 healthy volunteers at a mean age of $55.4 \pm 10.6$ years, and 50 patients with acute hemispheric ICS at a mean age of 58.4 \pm 9.7 years, hospitalized in the Second Clinic for Neurology Diseases with NICU at St. Marina University Hospital in Varna, for the period 2014-2015.

The studied patients were selected using respective inclusion and exclusion criteria. The clinical study includes detailed history, clinical and neurological examination at the time of hospitalization. The first notice of sudden onset of neurological symptoms is considered the starting point of a stroke. All patients were examined using a Siemens Somatom Definition CT at the Diagnostic Imaging Centre at St. Marina University Hospital in Varna, during the first 48 hours of the onset of clinical symptoms. CT findings were in line with ischemic stroke in the territory of the MCA, with the involvement of the IC being determined, respectively. For the purposes of our study, the patients were divided into groups according to the location of the stroke: patients with stroke in the
TRMCA without IC $-22.9 \%(\mathrm{n}=16)$, in the territory of the left middle cerebral artery (TLMCA) without IC $-21.4 \%(n=15)$ in the TRMCA with IC $-15.7 \%$ $(\mathrm{n}=11)$, and in the TLMCA with IC $11.4 \%(\mathrm{n}=8)$.

BP was measured using an automatic blood pressure cuff attached to an electronic monitor EDAN for continuous monitoring of systolic and diastolic blood pressure and heart rate in real time. The values of BP and HR were registered every minute within 10 minutes and the mean value of the indicators was calculated. The mean arterial pressure (MAP) value was calculated according to a universal formula: MAP $=[$ (systolic blood pressure + 2xdiastolic blood pressure) / 3]

Tests included in the Ewing battery were applied for the evaluation of cardiovascular autonomic function using a Hokanson ANS2000 (Hokanson, Inc; Bellevue, WA) device. The results of each test were defined as normal, borderline and abnormal, and autonomous disorders were classified as normal, mild, expressed, severe and atypical. The severity of the autonomic dysfunction was assessed according to the Ewing classification and the patients were divided into two groups: group 1 with normal or mild disorders in the cardiovascular autonomic function and group 2 with expressed, severe and atypical disorders.

\section{RESULTS}

Elevated BP values at admission (over 140 $\mathrm{mmHg}$ systolic and over $90 \mathrm{~mm} \mathrm{Hg}$ diastolic pressure) were observed in $56 \%$ of our patients, with significantly higher values reported for systolic blood pressure $(\mathrm{SBP})(\mathrm{p}<0.001)$, diastolic blood pressure (DBP) $(\mathrm{p}=0.001)$, MAP $(\mathrm{p}<0.001)$, and HR $(\mathrm{p}=0.027)$ compared to the control group.

The comparative analysis of the mean values of SBP among the studied groups showed a statistically significant difference between the values of SBP in the control group (120.00 $\mathrm{mm} \mathrm{Hg}$ ), and in the group of patients $(\mathrm{p}=0.003)$, the highest being in patients with stroke in TRMCA with and without IC (160.00mm Hg) (Fig. 1).

The t-test comparison of the mean values of SBP between the control group and the patients with RMCA with IC, showed a significant difference ( $t=-$ 4.31; $\mathrm{p}<0.001)$. A significant difference was observed between the mean values of SBP in the control group and in the patients with RMCA without IC ( $\mathrm{t}=-3.44$; 
Darina Georgieva-Hristova, Silva Andonova, Evgeniya Kalevska et al.

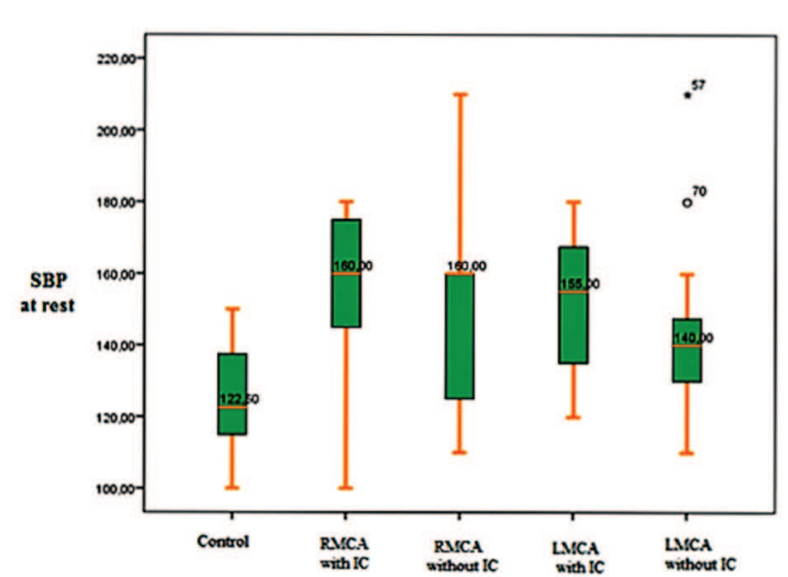

Figure 1. A comparative analysis of SBP values at restSBP at rest, Control, RMCA with IC, RMCA without IC, LMCA with IC, LMCA without IC

$\mathrm{p}=0.002)$, with LMCA with IC $(\mathrm{t}=-4.02 ; \mathrm{p}<0.001)$, and those with LMCA without IC $(\mathrm{t}=-3.08 ; \mathrm{p}=0.004)$.

The results from the ANOVA analysis showed a statistically significant difference in the values of DBP at rest in the investigated groups $(\mathrm{p}=0.016)$. The lowest values were in the control group $(80.00 \mathrm{~mm}$ $\mathrm{Hg}$ ), and the highest, in the RMCA with IC group (90.00 mm Hg) (Fig. 2).

The t-test showed the presence of a signifi-

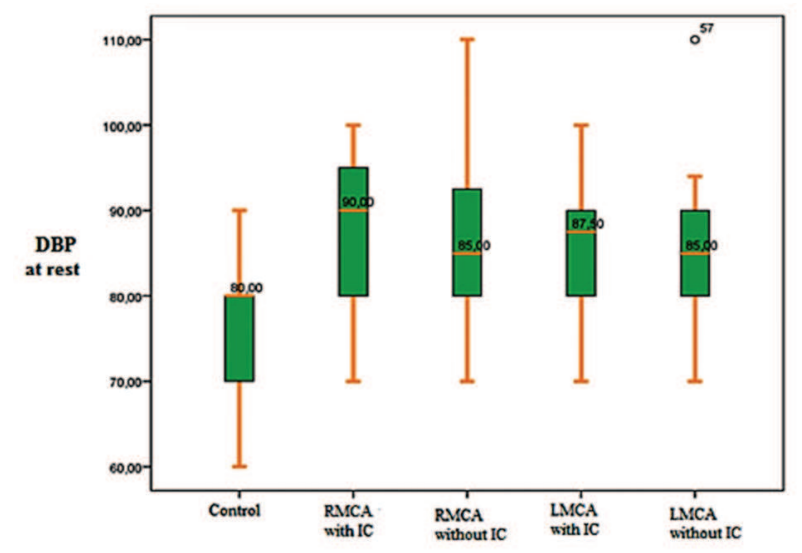

Figure 2. A comparative analysis of DBP values at rest $D B P$ at rest, Control, RMCA with IC, RMCA without IC, LMCA with IC, LMCA without IC

cant difference between the mean values of DBP of the control group and of patients with RMCA with IC $(t=-3.34 ; p=0.002)$. Statistically significant differences between the mean values of DBP at rest in the control group and the patients with RMCA without IC ( $85 \mathrm{~mm} \mathrm{Hg}),(\mathrm{t}=-2.72 ; \mathrm{p}=0.01)$, with LMCA with
IC $(87.5 \mathrm{~mm} \mathrm{Hg})(\mathrm{t}=-2.45 ; \mathrm{p}=0.021)$ and those with LMCA without IC $(85 \mathrm{~mm} \mathrm{Hg})(\mathrm{t}=-2.84 ; \mathrm{p}=0.008)$ were found.

The results of the ANOVA analysis showed a statistically significant difference in the values of MAP at rest in the studied groups $(\mathrm{p}=0.004)$, with the lowest values in the control group $(96.00 \mathrm{~mm}$ $\mathrm{Hg}$ ), and the highest in the RMCA and the LMCA with IC groups $(113.00 \mathrm{~mm} \mathrm{Hg}$ and $111.00 \mathrm{~mm} \mathrm{Hg}$, respectively) (Fig. 3.)

The t-test showed a significant difference be-

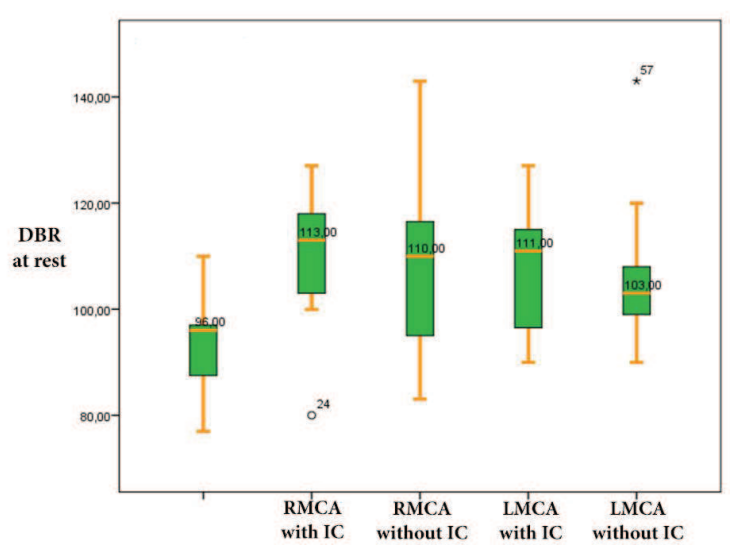

Figure 3. A comparative analysis of the MAP values at rest - MAP at rest, Control, RMCA with IC, RMCA without IC, LMCA with IC, LMCA without IC

tween the mean values of $\mathrm{BP}$ at rest in the control group and in the patients with RMCA with IC (113 $\mathrm{mm} \mathrm{Hg})(\mathrm{t}=-4.17 ; \mathrm{p}<0.001)$, with RMCA without IC $(110 \mathrm{~mm} \mathrm{Hg})(\mathrm{t}=-3.18 ; \mathrm{p}=0.003)$, with LMCA with IC $(111 \mathrm{~mm} \mathrm{Hg})(\mathrm{t}=-3.46, \mathrm{p}=0.002)$, and with LMCA without IC $(103 \mathrm{~mm} \mathrm{Hg})(\mathrm{t}=-3.24 ; \mathrm{p}=0.003)$.

The results of our study showed significantly higher values of HR in patients with ICS in comparison with the control group. The highest rates of $\mathrm{HR}$ were found in the patients who had ICS in the territory of the RMCA with IC (78 bpm) (Fig. 4).

The results of the t-test indicated a significant difference between the mean values of HR in the control group and in patients with RMCA with IC $(78 \mathrm{bpm})(\mathrm{t}=-2.51 ; \mathrm{p}=0.018)$. No difference was found in the mean values in the control group compared to subjects with RMCA without IC (74 bpm) ( $\mathrm{t}=-1.65$; $\mathrm{p}=0.108)$, with LSAM with IC (75.5 beats per min- 


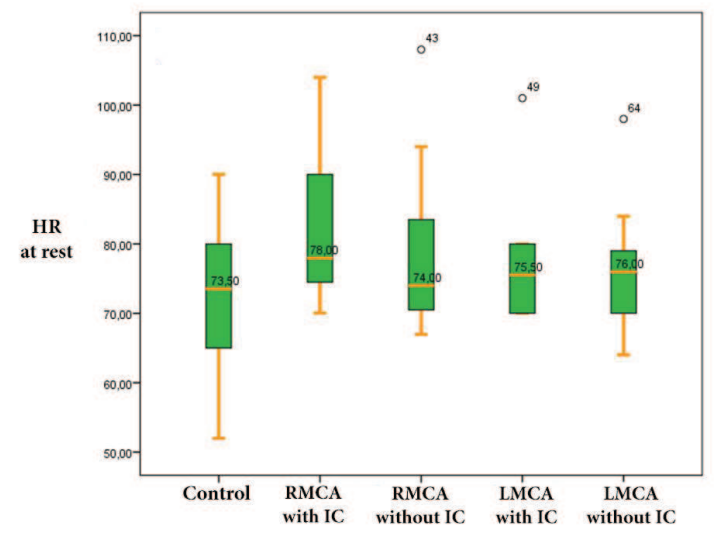

Figure 4. A comparative analysis of the $H R$ values at rest - HR at rest, Control, RMCA with IC, RMCA without IC, LMCA with IC, LMCA without IC

ute) $(\mathrm{t}=-1.21 ; \mathrm{p}=0.235)$ and with LMCA without IC (76 bpm) (t=-1.08; $\mathrm{p}=0.286)$.

Correlation between BP and HR, and the Severity of Autonomic Dysfunction

The participation of the ANS in the pathophysiology mechanism for the regulation of $\mathrm{BP}$ and $\mathrm{HR}$ suggests a correlation between the severity of the cardiovascular autonomous disorders and the values of BP and HR. The performed Student t-test showed that SBP at rest was significantly higher in the group of patients with severe impairment of the autonomic function $(152.8 \mathrm{~mm} \mathrm{Hg}$ ) compared to the values in the group of patients with mild autonomic disorders (139.0 mm Hg) ( $\mathrm{p}=0.036)$ (Fig. 5). The t-test showed a statistically significant difference in mean SBP values

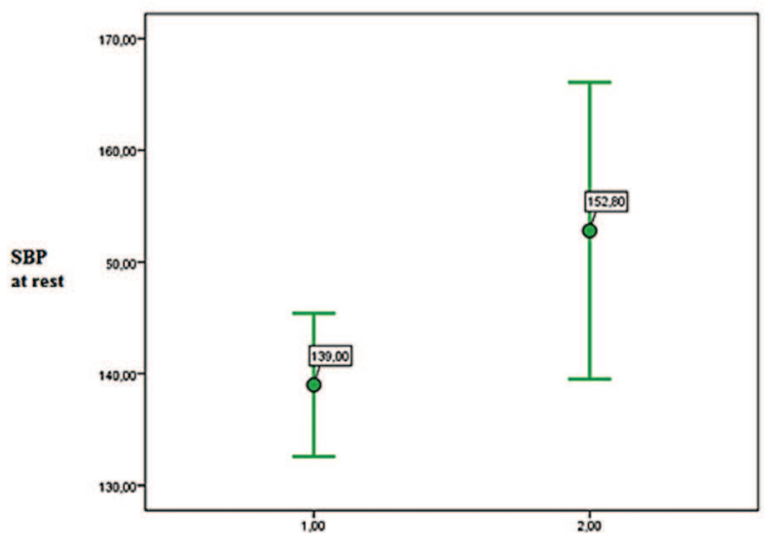

Figure 5. Comparison of SBP values at rest according to the severity of the autonomous disorders - 95\% CI 95\%, CI SBP at rest, Mild disorders, Serious disorders, Severity of autonomic dysfunction at rest according to the severity of the autonomous disorders ( $\mathrm{t}=-2.14 ; \mathrm{p}=0.036)$.

Unlike SBP at rest, the values of DBP showed no statistically significant difference between the two groups of patients. In mild autonomic disorders, the mean value of $\mathrm{DBP}$ at rest is $82.88 \mathrm{~mm} \mathrm{Hg}$, and in the severe ones $-85.65 \mathrm{mmHg}$ ( $\mathrm{p}>0.05)$.

The $t$-test showed no statistically significant difference between the two groups ( $t=-0.99$; $\mathrm{p}=0.323$ ). No significant difference was found in the values of MAP between the patients with mild autonomic disorders $(101.68 \mathrm{~mm} \mathrm{Hg})$ and those with severe autonomic dysfunction $(107.95 \mathrm{~mm} \mathrm{Hg})(\mathrm{t}=-1.66$, $\mathrm{p}=0.102$ ).

No significant difference was found in the mean values of HR between patients with mild autonomic disorders $(75.72 \mathrm{bpm})$ and those with a severe autonomic dysfunction (79.10 bpm) ( $\mathrm{t}=-1.19 ; \mathrm{p}=0.235)$.

\section{DISCUSSION}

There is a variability in SBP, DBP, MAP, and HR with a tendency towards their increase, as the initial higher values are most likely associated with activation of the sympathetic axis due to focal cerebral ischemia. Some authors have reported a higher incidence of elevated BP at admission, around 80\% (10).

Many researchers have published data for the possible fluctuation of BP in patients with stroke, and various studies have shown that the increase of $\mathrm{BP}$ values after stroke is transient (1) and associated with a poor outcome $(11,12,13,14)$. Elevated BP usually decreases spontaneously in the next few days after the stroke onset, without the application of antihypertensive therapy (15).

Our study data indicate the presence of a significant difference between the values of SBP, DBP and MAP between stroke patients and the control group, similar to the results of other authors (16). Patients with ischemic stroke and insular cortex damage present more often an increase of BP (17) and $\mathrm{HR}$ values, compared to those without involvement of the IC and those with left IC involvement. We report the most significantly elevated values in patients with ICS in the territory of the RMCA with involvement of IC, SBP $(\mathrm{t}=-4.31 ; \mathrm{p}<0.001), \mathrm{DBP}(\mathrm{t}=-3.34$; $\mathrm{p}=0.002)$, MAP $(\mathrm{t}=-4.17 ; \mathrm{p}<0.001)$, and HR $(\mathrm{t}=-2.51$; $\mathrm{p}=0.018)$, respectively. These results are probably as- 
Darina Georgieva-Hristova, Silva Andonova, Evgeniya Kalevska et al.

sociated with significantly elevated levels of catecholamines in lesions affecting the right IC (16) and the respective prevalence of the sympathetic activity. Our results support the key role of the right IC in the modulation of the sympathetic tone and can be explained by the evidence from the conducted electrophysiological tests for the presence of a higher percentage of sympatho-excitatory neurons in the right posterior IC $(18,19,20)$.

The results of the t-test showed a statistically significant difference in SBP in patients with mild and severe autonomic disorders, as SBP values were significantly higher in patients with severe disorders $(\mathrm{t}=-2.14 ; \mathrm{p}=0.036)$. Unlike SBP, the values of DBP, MAP, and HR did not show a significant difference between patients with mild and severe autonomous disorders $(p>0.05)$. These results can be explained with the pathogenetic mechanism of the impaired sympathetic-vagal modulation which, at rest, leads mainly to changes in the cardiac output and in the power of cardiac contractility (an increase in SBP, respectively), rather than to a change in the resistance of peripheral vessels.

In the acute phase of the ischemic stroke, the values of BP vary considerably and the clinical management is largely based on the mean values of $\mathrm{BP}$ at admission.

\section{CONCLUSION}

$\mathrm{BP}$ and $\mathrm{HR}$ variability with an increasing trend was observed in patients with acute hemispheric ischemic stroke, taking into account the significantly higher values compared with controls. A positive correlation between the values of SBP and the severity of $\mathrm{AD}$ was found.

\section{REFERENCES}

1. Qureshi A.I., Ezzeddine, M.A., Nasar, A. et al, Prevalence of elevated blood pressure in 563,704 adult patients with stroke presenting to the $\mathrm{ED}$ in the United States. Am J Emerg Med. 2007;25:32-38,

2. Qureshi AI. Acute hypertensive response in patients with stroke: pathophysiology and management. Circulation. 2008;118:176-187

3. Weiss A, Beloosesky Y, Kenett RS, Grossman EAm J Hypertens. Change in Systolic Blood Pressure During Stroke, Functional Status, and Long-Term
Mortality in an Elderly Population. Am J Hypertens. 2015 Jul 24. pii: hpvl18.

4. Nakano Y, Oshima T, Ozono R, Higashi Y, Sasaki S, Matsumoto T, et al. Non-dipper phenomenon in essential hypertension is related to blunted nocturnal rise and fall of sympatho-vagal nervous activity and progress in retinopathy. Auton Neurosci 2001;88:181-6.

5. Sander D, Winbeck K, Klingelhofer J, Etgen T, Conrad B. Prognostic relevance of pathological sympatheticactivation after acute thromboembolic stroke. Neurology 2001;57:833-8.

6. Carlberg, B., Asplund, K., Hagg, E. Factors influencing admission blood pressure levels in patients with acute stroke. Stroke. 1991;22:527-530.

7. Delgado-Mederos $\mathrm{R}^{1}$, Ribo M, Rovira A, Rubiera M, Munuera J, Santamarina E, et al., Prognostic significance of blood pressure variability after thrombolysis in acute stroke. Neurology. 2008 Aug 19;71(8):552-8.

8. Immink RV, van Montfrans GA, Stam J, Karemaker JM, Diamant M, van Lieshout JJ. Dynamic cerebral autoregulation in acute lacunar and middle cerebral artery territory ischemic stroke. Stroke. 2005;36(12):2595-600.

9. Ntaios, G., Lambrou, D., Michel, P. Blood pressure change and outcome in acute ischemic stroke: the impact of baseline values, previous hypertensive disease and previous antihypertensive treatment. J Hypertens. 2011;29:1583-1589.

10. De Raedt S, De Vos A, De Keyser J Autonomic dysfunction in acute ischemic stroke: an underexplored therapeutic area? J Neurol Sci. 2015 Jan 15;348(1-2):24-34.

11. Schrader J, Luders S, Kulschewski A, Berger J, Zidek W, Treib J,Einhaupl K, Diener HC, Dominiak P; Acute Candesartan Cilexetil Therapy in Stroke Survivors Study Group. The ACCESS Study: evaluation of Acute Candesartan Cilexetil Therapy in Stroke Survivors. Stroke. 2003;34:1699 - 1703.

12. Willmot M, Leonardi-Bee J, Bath PM. High blood pressure in acute stroke and subsequent outcome: a systematic review. Hypertension. 2004;43:18 -24.

13. Immink RV, van Montfrans GA, Stam J, Karemaker JM, Diamant M, van Lieshout JJ.Dynamic cerebral autoregulation in acute lacunar and middle cerebral artery territory ischemic stroke. Stroke. 2005;36(12):2595-600. 
Arterial blood pressure and heart rate response in patients with acute ischemic stroke correlation with the severity of cardiovascular ...

14. Geeganage C, Tracy M, England T, Sare G, Moulin T, Woimant F, et al. Relationship between baseline blood pressure parameters (including mean pressure, pulse pressure, and variability) and early outcome after stroke: data from the Tinzaparin in Acute Ischaemic Stroke Trial (TAIST). Stroke 2011;42:491-3

15. Hadjiev DI., Mineva PP. Elevated blood pressure management in acute ischemic stroke remains controversial: Could this issue be resolved? Med Hypotheses. 2013 Jan;80(1), 50-52.

16. Akıl E, Tamam Y, Akıl MA, Kaplan İ, Bilik MZ, Acar A, Tamam B. Identifying autonomic nervous system dysfunction in acute cerebrovascular attack by assessments of heart rate variability and catecholamine levels. J Neurosci Rural Pract. 2015; 6(2):145-50.

17. Meyer S, Strittmatter M,Fischer C, Georg T,Schmitz B. Lateralization in autonomic dysfunctionin ischemic stroke involving the insular cortex. Neuroreport 2004;15:357-61

18. Zhang ZH, Rashba S \& Oppenheimer SM. Insular cortex lesions alter baroreceptor sensitivity in the urethane-anesthetized rat. Brain Res 1998; 813, 73-81.

19. Oppenheimer, S.M., Cechetto, D. F., Hachinski, V.C. Cerebrogenic cardiac arrhythmias. Arch. Neurol., 47, 1990, 513-519.

20. Zhang ZH, Oppenheimer SM.

Characterization,distribution and lateralization of baroreceptor-related neurons in the rat insular cortex. Brain Res 1997, 760, 243-250. 